\title{
MOVIMENTO BROWNIANO: APLICAÇÃO EM ESTRATÉGIAS DE BUSCA
}

\section{BROWNIAN MOTION: APPLICATION IN SEARCHING STRATEGIES}

\author{
M. CARVALHO ${ }^{1}$, R. FALCÃO ${ }^{2}$, A. SIQUEIRA ${ }^{3}$ \\ ${ }^{1}$ Federal University of Viçosa, DEQ, Viçosa, MG, Brazil, matheus.d.carvalho@ufv.br \\ ${ }^{2}$ Federal University of São João Del-Rei, DEFIM, Ouro Branco, MG, Brazil, rfalcao@ufsj.edu.br \\ ${ }^{3}$ Federal University of Viçosa, DEQ, Viçosa, MG, Brazil, antonio.siqueira@ufv.br
}

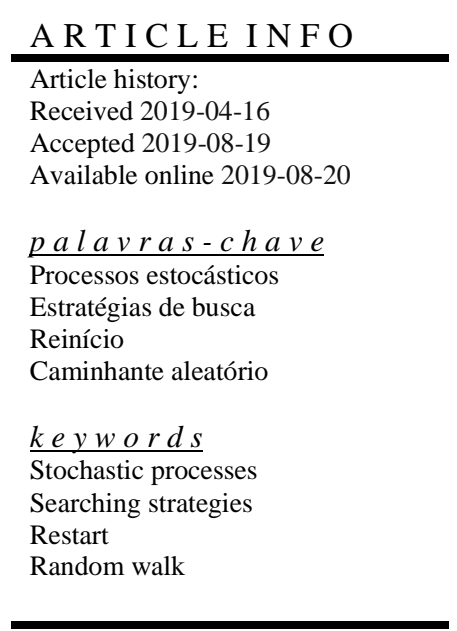

\begin{abstract}
A B S T R A C T
This article has elucidated information about Brownian Motion in the ring, something that is still little explored in the literature. In addition, the ideas offeed, metabolic rate and stochastic restart to the walker were added, features that have been gaining ground recently in the study of random processes. This paper structured partial differential equations governing this process for the immortal case of walker, and later found analytical solutions to these expressions. The representation of stationary state was also performed in graphical form, thus obtaining the distribution function of probability required. In order to briefly approach the walker in a deadly process, a graph was produced that presents the function between the number of steps taken by a walker before his death and his metabolic capacity.
\end{abstract}

\begin{abstract}
R E S U M O
Este artigo elucidou informações a respeito do movimento browniano no anel, algo ainda pouco explorado na literatura. Além disso, foram adicionadas as ideias de alimentação, taxa metabólica e reinício estocástico ao caminhante, características que vem ganhando espaço recentemente no estudo de processos aleatórios. Esse artigo realizou a estruturação das equações diferenciais parciais que regem tal processo para o caso de um caminhante imortal, além de posteriormente encontrar soluções analíticas para estas expressões. A representação do estado estacionário do caminhante também foi realizada na forma gráfica, obtendo assim as funções distribuição de probabilidade requeridas. Com o intuito de abordar brevemente o caminhante em um processo mortal, foi produzido um gráfico que apresenta a função entre o número de passos dados por um caminhante antes de sua morte e sua capacidade metabólica.
\end{abstract}




\section{INTRODUÇÃO}

Robert Brown, botânico inglês, observou no ano de 1828 um movimento desordenado de grãos de pólen imersos numa solução (Brown, 1828). Esse comportamento posteriormente adquiriu a denominação de movimento Browniano, em homenagem ao botânico. A explicação formal para esse movimento errático permaneceu um mistério por quase um século. Devido à curiosidade dos cientistas da época, diversas características experimentais foram coletadas durante o século XIX (Gouy, 1888). Dentre as características observadas pode-se citar o aumento da intensidade do movimento de acordo com a diminuição da viscosidade da solução ou do tamanho das partículas em suspensão, ou ainda com o aumento da temperatura do meio.

No ano de 1905 Albert Einstein apresentou uma explicação formal para o movimento misterioso (Einstein, 1905). Sua explicação se baseava em duas hipóteses. A primeira, assume que o movimento do grão de pólen é produzido por frequentes colisões com as moléculas de solvente. A segunda hipótese relata que o complexo movimento das moléculas de solvente pode ser descrito probabilisticamente em termos de colisões independentes. Este estudo representou um grande avanço para diversos campos da física e da química, entre eles pode-se citar a cinética de reações e a mecânica estatística.

O ramo dos processos estocásticos é responsável pelo estudo de eventos que possuem algum tipo de aleatoriedade associada. Dessa forma, são eventos definidos em termos probabilísticos e não de uma maneira exata. Entre os termos mais empregados nesse ramo cita-se a função densidade de probabilidade, função distribuição cumulativa e os momentos estatísticos (Hsu, 2014). A função densidade de probabilidade é uma maneira de quantificar a probabilidade de uma determinada variável aleatório adquirir um dado valor ou estar num certo intervalo. A Equação 1 apresenta a principal propriedade desta função.

$P[a \leq x \leq b]=\int_{a}^{b} f(x) d x$

A propriedade descrita pela Equação 1 representa que a probabilidade da variável aleatória $x$ estar entre os valores $a$ e $b$ é igual a integral da função densidade de probabilidade, simbolizada por $f(x)$. Dado que a probabilidade da ocorrência de um evento é um valor entre 0 e 1, quando se realiza a integração para todos os valores possíveis de $x$, obtém-se que:

$$
P[-\infty \leq x \leq \infty]=\int_{-\infty}^{\infty} f(x) d x=1
$$

A função de distribuição cumulativa é definida como a probabilidade de determinada variável aleatória $x$ possuir um valor inferior a uma quantidade $b$. Dessa forma, pode ser relacionada diretamente com a função densidade de probabilidade pelas Equações 3 e 4. Nas quais, $F(x)$ representa a função de distribuição cumulativa.

$$
\begin{aligned}
& F(x)=P[x \leq b]=\int_{-\infty}^{b} f(x) d x \\
& f(x)=\frac{d F(x)}{d x}
\end{aligned}
$$

Momentos estatísticos são grandezas obtidas através de cálculos com a função densidade de probabilidade, estes têm a finalidade de definir as características dessas distribuições. O enésimo momento estatístico de uma determinada distribuição $f(x)$ é representado pelo símbolo $E\left[X^{n}\right]$. O valor dessa grandeza é obtido pela aplicação da Equação 5, dado que $n$ é um número natural.

$$
E\left[X^{n}\right]=\int_{-\infty}^{\infty} x^{n} f(x) d x
$$

O principal momento estatístico utilizado neste trabalho é o de primeira ordem, este representa o valor médio de uma variável aleatória. A simbologia deste momento é dada por $E[x]$ e tem valor determinado pela Equação 6 .

$$
E[x]=\int_{-\infty}^{\infty} x \cdot f(x) d x
$$

O presente texto utiliza de todos esses conceitos do ramo de processos estocásticos, com o objetivo de encontrar propriedades que se apliquem tanto a processos aleatórios microscópicos e macroscópicos. Entre os processos microscópicos tem-se o movimento desordenado de partículas em um fluido, como citado anteriormente. Além disso, a aleatoriedade está presente nas reações químicas e nos processos de transferência de massa e calor (Smith e Grima, 2016). Processos aleatórios macroscópicos são comuns na natureza, principalmente ao se tratar do reino animal. A busca por alimento entre os animais é um exemplo clássico, criando a relação presa-predador.

Devido a característica inteligente ou instintiva dos animais, as pesquisas que abordam a busca por algum recurso ou alimento tem adicionado parâmetros para tornar esses modelos mais próximos da realidade. Entre estes parâmetros cita-se a capacidade metabólica, taxa de renovação de recurso e o reinício estocástico (Evans and Majumdar, 2011; Chupeau et al., 2016). A capacidade metabólica, representada por $S$, indica o número de passos que um caminhante pode realizar sem morrer por inanição. O termo caminhante pode ser substituído por animal, sem perder seu significado.

A taxa de renovação de recurso representa o tempo 
necessário para o alimento reaparecer na posição em que foi consumido, este parâmetro é designado por $R$. O reinício estocástico é responsável pelo retorno casual do caminhante a sua posição inicial. Este mecanismo visa incluir uma espécie de estratégia de busca do caminhante, de forma que ele consiga estar sempre na região em que tem maior probabilidade de encontrar alimento. Dado que após um longo tempo de caminhada, este pode se encontrar muito distante de seu alvo.

A criação de estratégias de busca são ações recorrentes no nosso cotidiano. Na procura por um objeto perdido é comum que após um certo tempo de busca sem sucesso, a pessoa retorne a sua posição inicial e reinicie a procura a partir desse ponto de uma forma mais detalhada. Além disso, esse ponto inicial geralmente é aquele em que a pessoa considera possuir maior probabilidade de encontrar $\mathrm{o}$ objeto, seja por intuição ou algum tipo de memória.

O presente artigo visa estudar as funções densidade de probabilidade e cumulativas que representem a probabilidade de encontro entre dois caminhantes. Este estudo será realizado para uma geometria circular, na qual haverá o reinício dos caminhantes para suas respectivas posições iniciais após o encontro. Nas condições supracitadas, esta é uma situação ainda inexplorada na literatura.

\section{REVISÃO DA LITERATURA}

Estudos recentes vêm abordando o estudo de caminhantes aleatórios em diversas circunstâncias, nas quais são incluídos novos parâmetros. Outra alternativa é a variação de parâmetros abordados anteriormente, além de alterar a geometria e o número de caminhantes presentes. Esta seção tem por sua vez a função de apresentar alguns desses estudos.

Fisher (1984) desenvolveu um dos primeiros trabalhos a respeito da análise do encontro de caminhantes aleatórios. Este estudo envolveu redes com dimensões infinitas, nas quais há o movimento de $p$ caminhantes aleatórios independentes. Este artigo abordou redes com até três dimensões.

Gabel et al. (2012) estudaram a probabilidade de sobrevivência de um cordeiro diante da presença de $N$ leões a sua busca. Este estudo foi realizado para um ambiente infinito e unidimensional, no qual o cordeiro se salva ao alcançar determinado ponto da rede. Esse ponto ficou denominado no trabalho como céu. Resultados analíticos forma encontrados para o caso com um leão e infinitos leões.

Evans e Majumdar (2011) incluem no seu estudo a possibilidade do animal retornar para sua posição inicial a qualquer momento da sua caminhada. Esta característica é denominada como reinício estocástico, sendo que esse processo de retorno tem o objetivo de maximizar a possibilidade do caminhante encontrar alimento. Os pesquisadores encontraram a taxa ótimo de reinício para um caminhante a procura de alimento numa rede contínua e unidimensional.

Redner e Bénichou (2014) estudam a dinâmica de um sistema no qual um homem com difusividade $D_{M}$ é atacado por mosquitos de difusividade $D_{m}$. A cada encontro entre o mosquito e o homem, este último indivíduo recebe uma picada. A cada evento dessa natureza a difusividade do homem decai uma certa quantidade fixa, enquanto a difusividade do mosquito permanece inalterada. Após esses eventos, o homem e o mosquito são separados por última distância $a$. O homem morre após sua difusividade ser anulada. Este trabalho encontrou as funções densidade de probabilidade que regem o movimento do indivíduo atacado.

Chupeau et al. (2016) adotaram no seu estudo parâmetros como capacidade metabólica e taxa de renovação de recurso. O sistema estudado aborda um caminhante aleatório, que pode ser visto como um coelho, este caminha numa rede unidimensional e discreta coberta por alimento. $\mathrm{O}$ caminhante dá passos de tamanhos unitários em intervalos de tempo fixos. Ao se alimentar do recurso o animal tem sua necessidade metabólica satisfeita. Este artigo realizou a divisão do sistema em três regimes, de forma que o caminhante pode ser mortal independente da taxa de renovação de recurso, mortal dependendo da taxa de renovação ou imortal. Estes três regimes são funções da taxa metabólica e da renovação do alimento.

Falcão e Evans (2016) abordam as probabilidades de encontro entre duas partículas com movimento aleatório numa rede unidimensional, de forma que há o reinício para uma posição fixa após o encontro. As partículas sofrem uma espécie de $d r i f t$, que pode ser analisada como uma força de atração desses corpos.

Smith e Grima (2016) estudam a reação química reversível entre dois compostos $A$ e $B$ que geram um produto $C$. As partículas de reagente se difundem por uma caminhada aleatória num meio unidimensional. Ao se encontrarem, o processo reacional acontece e estas partículas se ligam. Essa ligação permanece por um intervalo de tempo definido, porém após esse intervalo, essa molécula $C$ se decompõe nos reagentes $A$ e $B$. Nesse momento que o processo se reinicia. Este artigo encontrou as funções densidade de probabilidade que descrevem o movimento desses corpos.

Belan S. (2018) descreve a etapa de reinício com uma aproximação dos voos de Levy. Os voos de Levy são representantes de passos que atingem distâncias acima da média percorrida pelos passos usuais que ocorrem num sistema. Esses eventos são consideravelmente raros quando se analisa o número total de passos, mas não podem ser negligenciados (Marques e Chauí-Berlink, 2014). Dessa forma, o reinício é visto como uma etapa anômala que acontece de tempos em tempos num sistema.

Em relação à geometria circular, a literatura apresenta resultados para caminhadas sem reinício ou qualquer probabilidade do caminhante finalizar sua busca por falta de recurso. A solução amplamente conhecida é a distribuição de Jacobi Theta, que nesse caso representa a probabilidade do caminhante estar em determinada posição em certo instante de tempo (Widder, 1975). As funções de Jacobi Theta são uma importante classe de funções, que surgem na resolução de problemas de transferência de calor e massa em ambiente simétrico. 


\section{RESULTADOS E DISCUSSÃO}

Esta seção apresenta os resultados obtidos durante a execução desse estudo e as respectivas discussões. Primeiramente esta seção apresenta uma análise discreta do sistema a ser abordado, de forma a explicar e equacionar a dinâmica envolvida. Devido à dificuldade de encontrar uma solução que obedeça às equações geradas, realizasse o equacionamento na forma contínua. Para esse equacionamento são encontradas soluções por duas metodologias, tanto por separação de variáveis, quanto pelo método de imagem. Pelo primeiro método são obtidas algumas propriedades que caracterizam o sistema, entre elas tem-se o tempo de vida médio do caminhante e a função distribuição cumulativa para a sobrevivência.

$\mathrm{O}$ método de imagem se mostrou uma metodologia fundamental para resolução do problema com a inclusão do reinício, devido a maior eficiência na inversão da transformada de Laplace associada. Um estado estacionário é encontrado para o processo, de modo que este é representado por uma equação e na forma gráfica. A seção finaliza com a análise do processo para um caminhante mortal. Numericamente obtém-se um gráfico que relaciona o número de passos dados por um caminhante antes de morrer e sua respectiva taxa metabólica. Uma curva de regressão exponencial com seus devidos parâmetros é obtida para esses dados, finalizando o estudo proposto.

\subsection{Análise discreta}

A descrição da dinâmica do modelo no formato discreto auxilia no entendimento do problema, por ser uma abordagem mais intuitiva. $\mathrm{O}$ ambiente da caminhada será um anel com $L$ sítios, sendo estes os locais possíveis para o posicionamento dos caminhantes. No interior desta geometria existem 2 caminhantes aleatórios, estes estarão inicialmente distantes $m_{0}$ sítios entre si. Esta última grandeza deve ser um número par. E além disso, o número total de sítios deve ser múltiplo de 4 , dessa maneira os caminhantes podem ocupar o mesmo sítio concomitantemente durante suas trajetórias. Para fins didáticos, um destes sujeitos será representado por um leão e o outro sujeito por um cordeiro. Estes dois caminhantes se movimentam numa trajetória randômica até se encontrarem, por analogia, o leão se alimenta do cordeiro nesse caso. A figura 1 representa a configuração do sistema.

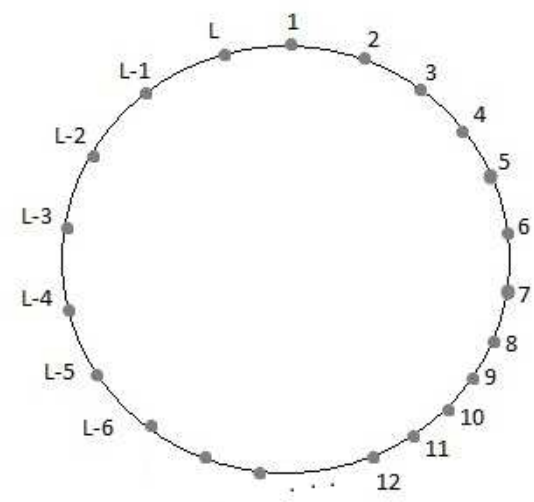

Figura 1 - Representação esquemática do ambiente abordado no problema.
A metodologia aplicada para o equacionamento do sistema envolve analisar a variação da distância mínima entre os caminhantes com o passar do tempo. Dada que essa distância seja de $x$ sítios num dado instante de tempo $t$, a evolução desse sistema possui a seguinte dinâmica no espaço discreto.

$$
\begin{aligned}
& C(x, t)=\frac{1}{4} C(x-2, t-1)+\frac{1}{2} C(x, t-1)+ \\
& \frac{1}{4} C(x+2, t-1), \forall x \neq 0 \vee x \neq 2 \vee x \neq \frac{L}{2}
\end{aligned}
$$

Na Equação $7, C(x, t)$ representa a probabilidade das distâncias entre os caminhantes ser $x$ no tempo $t$. Já a Equação 8, se refere ao movimento um único caminhante. Nesta expressão $P(x, t)$ é a probabilidade do caminhante estar na posição $x$ no tempo $t$. A Equação 9 é a representação da Equação 8 em função do tempo t-2.

$$
\begin{aligned}
& P(x, t)=\frac{1}{2} P(x-1, t-1)+\frac{1}{2} P(x+1, t-1) \\
& P(x, t)=\frac{1}{4} P(x-2, t-2)+\frac{1}{2} P(x, t-2)+ \\
& \frac{1}{4} P(x+2, t-2)
\end{aligned}
$$

A comparação entre as Equações 7 e 9, levam a constatação de que o processo ocorre de maneira acelerada para $C(x, t)$. De forma que o movimento equivalente de $P(x, t)$ necessita de 2 instantes de tempo para acontecer, em contra partida, para $C(x, t)$ necessita de apenas 1 . Este efeito acontece devido a contribuição do movimento dos dois caminhantes na Equação 7, enquanto na Equação 8 envolve apenas um.

Devido a característica finita do sistema e a sua simetria, os caminhantes podem ficar no máximo a $\frac{L}{2}$ sítios de distância. Em função disso, a dinâmica nesse ponto se comporta de uma maneira diferente:

$C\left(\frac{L}{2}, t\right)=\frac{1}{2} C\left(\frac{L}{2}, t-1\right)+\frac{1}{2} C\left(\frac{L}{2}-2, t-1\right)$

O ponto de encontro entre os caminhantes possui uma característica geralmente denominada na literatura como absorvente (Redner, 2001). Essa nomenclatura tem como o objetivo caracterizar esse ponto como um sumidouro. Exemplos clássicos para representar essa característica são o de membranas absorvedoras de soluto ou superfícies reativas. Nesses exemplos o composto é absorvido ou consumido no momento do contato físico. Este conceito para o sistema estudado é entendido como a morte do cordeiro ao se encontrar com o leão. As Equações 11 e 12 representam o 
comportamento do sistema próximo ao sumidouro.

$$
C(0, t)=C(2, t-1)
$$

$$
C(2, t)=\frac{1}{2} C(2, t-1)+\frac{1}{2} C(4, t-1)
$$

Em geral, problemas com abordagem discreta são resolvidos pelo método de função geratriz (PTASZYNSKI, 2018). Porém, este processo é bem-sucedido apenas para casos de caminhadas mais simples, nas quais não há por exemplo a ideia de reinício. Essa limitação ocorre principalmente em função da dificuldade na etapa de inversão das equações geradas (Wilf, 1992). Devido a esse fato, optou-se por trabalhar com o problema numa abordagem contínua, como será realizado na próxima subseção.

\subsection{Análise do sistema contínuo sem reinício}

Nesta subseção o problema sistematizado anteriormente da maneira discreta, passa a ser abordado na sua forma contínua, através de uma equação diferencial parcial (EDP). Para obtenção de uma solução única, são utilizadas duas condições de contorno e uma condição inicial. São necessárias duas condições de contorno em função da derivada espacial na EDP ser de segunda ordem.

O sistema de equações é solucionado pela aplicação de dois métodos conhecidos na literatura: separação de variáveis (Boyce e Diprima, 2010) e método de imagem (Redner, 2001).

Separação de variáveis: O problema abordado tratase de apenas um leão e um cordeiro difundindo no anel contínuo, no qual o leão possui difusividade $D_{l}$ e o cordeiro difusividade $D_{2}$. Como se tratam de caminhantes aleatórios, a caminhada destes obedece a equação de difusão, fato bastante conhecido na literatura (Sokolov e Krafter, 2005). Como abordado anteriormente, a variação da distância mínima dos caminhantes se comporta como a variação da posição dos caminhantes, porém de forma acelerada. Dessa forma, a função $C(x, t)$ obedece a Equação 13 , na qual $D$ é igual a soma das difusividades dos dois caminhantes, como constatado pela análise das Equações 7 e 9.

$\frac{\partial C(x, t)}{\partial t}=D \frac{\partial^{2} C(x, t)}{\partial x^{2}}$

Este problema envolve duas condições de contorno, sendo uma absorvente e a outra refletora. A condição refletora se deve ao fato dos caminhantes não poderem ficar a uma distância superior a $\frac{L}{2}$. Dessa forma, essa fronteira se comporta como uma barreira, a qual os caminhantes não podem transpor. Analogamente a um processo de transferência de massa num tubo, essa fronteira seria como um tampão, que impede a passagem do soluto. Estas condições estão expressas nas Equações 14 e 15:
$C(0, t)=0$

$\frac{\partial C\left(\frac{L}{2}, t\right)}{\partial x}=0$

Estas equações são conhecidas na literatura como condições de Dirichlet e Neumann (Boyce e Diprima, 2010), respectivamente. Outra denominação usual é a de condições de primeira e segunda espécie. Como condição inicial temse que a distância entre os caminhantes é de $m_{0}$, como se trata de uma condição pontual, esta é representada pela função Delta de Dirac (Lutzer, 2007):

$C(x, 0)=\delta\left(x-m_{0}\right), 0 \leq m_{0} \leq \frac{L}{2}$

A solução deste problema já é clássica na literatura, ela está presente nos textos de Smith e Grima (2016) e Boyce e Diprima (2010). A resolução pelo método de separação de variáveis resulta na Equação 17:

$C(x, t)=\sum_{n=1}^{\infty} \frac{4}{L} \operatorname{sen}\left(\frac{(2 n-1) \pi m_{0}}{L}\right) \operatorname{sen}\left(\frac{(2 n-1) \pi x}{L}\right) e^{-\frac{(2 n-1)^{2} \pi^{2} D t}{L^{2}}}$

Com posse da função $C(x, t)$, torna-se possível descobrir algumas característica do modelo equacionado. Uma delas é a probabilidade, $M(t)$, do leão se alimentar até o tempo $t$, que é dada pela Equação 18. Essa expressão é obtida pela exclusão de todas as trajetórias que ainda estão presentes no anel, ou seja, que ainda não atingiram a fronteira absorvente. Uma analogia seria o processo de transferência de calor numa barra metálica, que possui com uma borda com a temperatura fixa de $0 \mathrm{~K}$. Essa borda se comporta como um sumidouro de energia, devido ao fato de possuir a temperatura mínima possível na natureza e ao fato da transferência de calor ser movida pela diferença de temperatura. Para quantificar a quantidade de energia que foi absorvida, bastaria realizar a integração da capacidade calorífica do metal multiplicada pela temperatura em cada ponto. E posteriormente subtrair da quantidade inicial de energia presente na barra.

$$
\begin{aligned}
& M(t)=1-\int_{0}^{\frac{L}{2}} C(x, t) d x \\
& =1-\sum_{n=1}^{\infty} \frac{4}{(2 n-1) \pi} \operatorname{sen}\left(\frac{(2 n-1) \pi m_{0}}{L}\right) e^{-\frac{(2 n-1)^{2} \pi^{2} D t}{L^{2}}}
\end{aligned}
$$

Uma propriedade da função $M(t)$ é que ela tende a 1 quando o tempo tende ao infinito, se comportando como uma distribuição cumulativa. Dessa forma, os dois caminhantes tendem sempre a se encontrar, independentemente do tamanho do anel. A circunferência do 
anel influência apenas no tempo em que o encontro irá acontecer, como mostra a Equação 18. Como a probabilidade do leão se alimentar e do cordeiro sobreviver são exemplos excludentes. A probabilidade de sobrevivência do cordeiro, denotada por $S(t)$, pode ser calculada como o complementar de $M(t)$, sendo representada pela Equação 19 .

$S(t)=1-M(t)$

$=\sum_{n=1}^{\infty} \frac{4}{(2 n-1) \pi} \operatorname{sen}\left(\frac{(2 n-1) \pi m_{0}}{L}\right) e^{-\frac{(2 n-1)^{2} \pi^{2} D t}{L^{2}}}$

A partir da Equação 19 nota-se que a probabilidade de sobrevivência do cordeiro decresce exponencialmente com o tempo. Outra característica que pode ser calculada é o tempo médio de sobrevivência do cordeiro. Este é obtido pela definição de primeiro momento estatístico, esta grandeza será representada por $\langle t\rangle$. Porém, o cálculo do tempo médio deve ser realizado a partir da função densidade de probabilidade, representada por $f(t)$. A função $f(t)$ deve ser obtida a partir da derivação da função distribuição cumulativa, $M(t)$, como apresenta a Equação 4. Dessa forma:

$$
\begin{aligned}
& f(t)=M^{\prime}(t) \\
& =\sum_{n=1}^{\infty} \frac{4(2 n-1) \pi D}{L^{2}} \operatorname{sen}\left(\frac{(2 n-1) \pi m_{0}}{L}\right) e^{-\frac{(2 n-1)^{2} \pi^{2} D t}{L^{2}}}
\end{aligned}
$$

Com posse da $f(t)$, o tempo médio é encontrado pela aplicação da Equação 6.

$$
\begin{aligned}
& <t>=\int_{0}^{\infty} t f(t) d t \\
& =\sum_{n=1}^{\infty} \frac{4 L^{2}}{(2 n-1)^{3} \pi^{3} D} \operatorname{sen}\left(\frac{(2 n-1) \pi m_{0}}{L}\right)
\end{aligned}
$$

Considerando que o leão possui uma taxa metabólica $t_{M}$, de modo que, se o predador ficar sem se alimentar por um tempo superior a este, ele morrerá. A probabilidade do leão morrer será dada por $S\left(t_{M}\right)$, de forma que:

$S\left(t_{M}\right)=\sum_{n=1}^{\infty} \frac{4}{(2 n-1) \pi} \operatorname{sen}\left(\frac{(2 n-1) \pi m_{0}}{L}\right) e^{-\frac{(2 n-1)^{2} \pi^{2} D t_{M}}{L^{2}}}$

Todas essas características são utilizadas para melhorar o entendimento do sistema estudado. Porém o estudo com a aplicação do reinício por esta metodologia se apresentou inviável, devido à dificuldade na inversão das transformadas de Laplace obtidas. Dessa maneira, o problema também foi solucionado por uma outra metodologia, denominada método de imagem, a qual apresentou melhores característica analíticas para avançar no estudo.
Método de imagem: O método trata-se de uma metodologia de resolução de equações diferenciais parciais, geralmente utilizada no estudo de caminhantes aleatórios (Redner, 2001; Gabel et al., 2012). Porém pode ser aplicada para outras abordagens que envolvam equações similares, como na transferência de massa e calor. $O$ primeiro passo consiste na resolução da equação de difusão em um sistema infinito, assim depois se adiciona as condições de contorno com a utilização de gaussianas e antigaussianas a solução (Redner, 2001).

Para a resolução de um problema de difusão num meio infinito se faz necessário apenas a condição inicial, devido à ausência de fronteiras. Dessa forma, a solução é dada pela função $C_{0}(x, t)$, que deve obedecer as seguintes Equações:

$$
\begin{gathered}
\frac{\partial C_{0}(x, t)}{\partial t}=D \frac{\partial^{2} C_{0}(x, t)}{\partial x^{2}} \\
C_{0}(x, 0)=\delta\left(x-m_{0}\right)
\end{gathered}
$$

Este problema já possui solução conhecida na literatura (Redner, 2001), esta é obtida através da aplicação da transformada de Fourier e de Laplace, sendo representada pela Equação 25:

$$
C_{0}(x, 0)=\frac{1}{\sqrt{4 \pi D t}} e^{-\frac{\left(x-m_{0}\right)^{2}}{4 D t}}
$$

Este resultado não representa a solução do problema desejado, sendo necessário utilizar o método de imagem para formar as barreiras refletoras e absorventes. Para criar barreiras absorventes se utiliza antigaussianas a mesma distância do ponto zero, para criar barreiras refletoras se utiliza gaussianas positivas simétricas a outra extremidade. A Equação 26 apresenta a solução para um caso particular, no qual a distância $m_{0}$ é igual a $\frac{L}{2}$ e o comprimento total do anel é de $2 \mathrm{~L}$.

$$
\begin{aligned}
& C(x, t)=\frac{1}{\sqrt{4 \pi D t}} \sum_{n=-\infty}^{n=\infty}(-1)^{n} e^{\frac{-(x-(4 n+1) L)^{2}}{4 D t}} \\
& +\sum_{n=-\infty}^{n=\infty}(-1)^{n+1} e^{-\frac{-(x+(4 n+1) L)^{2}}{4 D t}}
\end{aligned}
$$

As figuras 2 e 3 apresentam como é a disposição das gaussianas e antigaussianas nessa solução. 


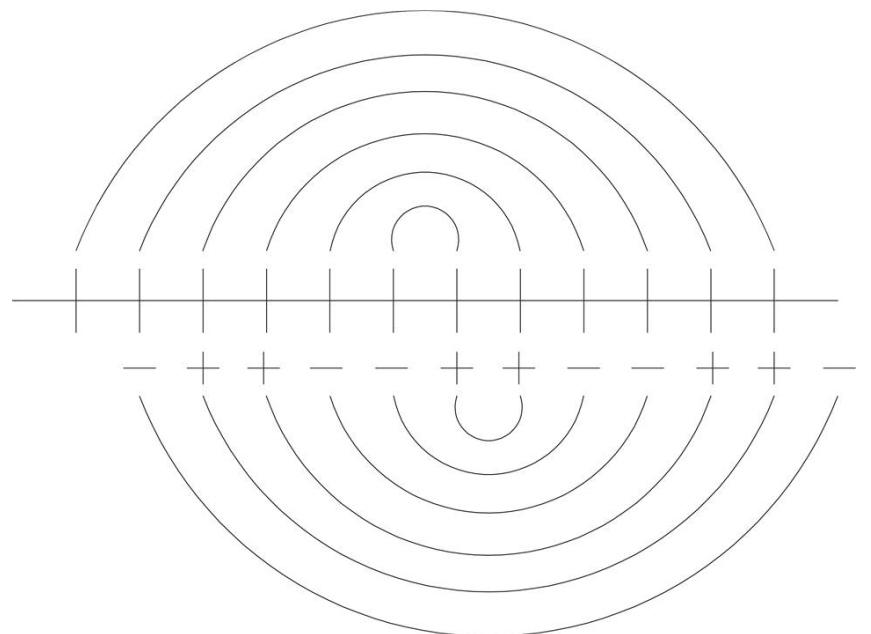

Figura 2 - Alternância das gaussianas e antigaussianas, representadas por sinais positivos e negativos.

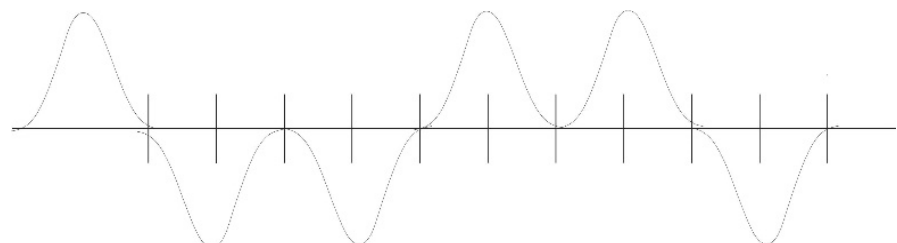

Figura 3 - Alternância das gaussianas e antigaussianas.

Com posse da solução do caso sem reinício, tornase possível encontrar a equação para o sistema com reinício. A metodologia aplicada está presente na seguinte subseção.

\subsection{Análise do sistema contínuo com reinício}

O sistema abordado nessa seção possui características similares ao anterior, como a de um leão imortal e movimento dos dois caminhantes num anel. Porém, neste caso inclui-se o retorno do leão e do cordeiro para suas posições iniciais imediatamente após o encontro. Essa posição inicial pode ser vista como uma espécie de toca, a qual é imediatamente abandonado após a etapa de retorno.

A equação que se deseja encontrar nesse caso, também é aquela que descreve a probabilidade da distância entre o leão e o cordeiro ser de $x$ no tempo $t$, denotada aqui por $C_{l}(x, t)$. Essa função obedece a mesma equação diferencial de um processo de transferência de massa de um soluto que foi gotejado num ponto de um tubo. De forma que todo o soluto que é absorvido por uma membrana na borda do tubo, é deslocado para a posição inicial em que foi gotejado. Sendo este um tubo fechado na outra extremidade. A equação diferencial parcial que governa esse fenômeno é dada pela seguinte expressão.

$\frac{\partial C_{1}(x, t)}{\partial t}=D \frac{\partial^{2} C_{1}(x, t)}{\partial x^{2}}+h(t) \delta\left(x-m_{0}\right)$

A diferença entre as Equações 23 e 27 é a adição de termo, este é o responsável pelo comportamento de reinício. Este termo é o resultado da multiplicação do fluxo na extremidade absorvente, representado por $h(t)$, e a função Delta de Dirac na posição inicial da difusão. A função Delta de Dirac garante que todo o fluxo será destinado para a posição inicial. A função $h(t)$ pode ser representada pela derivada primeira em relação a posição da função $C(x, t)$ na posição 0 , exatamente por ser representativa do fluxo nessa região.

$h(t)=\frac{\partial C_{1}(x, t)}{\partial x} ; x=0$

As duas condições de contorno e a condição inicial desse processo são as mesmas do evento sem reinício, variando somente a EDP envolvida.

$C_{1}(0, t)=0$

$\frac{\partial C_{1}\left(\frac{L}{2}, t\right)}{\partial x}=0$

$C(x, 0)=\delta\left(x-m_{0}\right)$

A partir da solução homogênea obtida pelo método de imagem, Equação 26, é possível obter $C_{l}(x, t)$ pela aplicação da Equação 32 (Falcão e Evans, 2016). Esta equação tem o objetivo de adicionar a contribuição do fluxo, a cada instante de tempo $t$, sobre a função de distribuição homogênea.

$C_{1}(x, t)=C(x, t)+\int_{0}^{t} C\left(x, t-t^{\prime}\right) h\left(t^{\prime}\right) d t^{\prime}$

Na equação 32, o primeiro termo do lado esquerdo representa a contribuição das trajetórias que não sofreram reinício. Enquanto o segundo termo representa a contribuição das trajetórias que sofreram a última renovação no tempo $t^{\prime}$.

Com o intuito de encontrar a função $h(t)$ apresentada pela Equação 28, aplica-se a transformada de Laplace na Equação 27. Além da derivação em relação a $x$, algebrismos e a expressão para convergência de séries geométricas. Assim encontra-se que:

$$
\begin{aligned}
& h(s)=\frac{D C_{x}(0, s)}{1-D C_{x}(0, s)} \\
& =\frac{\cosh \left(\sqrt{\frac{s}{D}} L\right)}{2 \operatorname{senh}\left(\frac{3 L}{2} \sqrt{\frac{s}{D}}\right) \cosh \left(\frac{L}{2} \sqrt{\frac{s}{D}} L\right)}
\end{aligned}
$$


A expressão para convergência de séries geométricas utilizada é dada pela expressão 34 (Stewart, 2017). Na qual, $s_{n}$ representa a soma dos $n$ primeiros termos, $a_{0}$ é o primeiro termo e $r$ é denominado como razão da série.

$\lim _{n \rightarrow \infty} s_{n}=\lim _{n \rightarrow \infty}\left(a_{0}+a_{0} r+a_{0} r^{2}+\cdots+a_{0} r^{n}\right)=$

$\frac{a_{0}}{1-r} ;|r|<1$

Para a inversão da equação 33 e encontrar a função $h(t)$, utiliza-se do teorema de convolução (Boyce e Diprima, 2010). Este teorema é enunciado na Equação seguinte.

$L^{-1}[F(s) \cdot G(s)]=\int_{0}^{t} f(\tau) g(t-\tau) d \tau$

Esta é uma equação que possibilita a inversão do produto da transformada de duas funções conhecidas. Com o auxílio de uma tabela de transformadas integrais (Erdélyi, 1954) e separando o lado direito da Equação $33 \mathrm{em}$ duas parcelas, encontra-se os resultados aplicáveis a esse problema.

$L^{-1}\left[\frac{\cosh (x \sqrt{s})}{\operatorname{senh}(l \sqrt{s})}\right]=-\frac{1}{l} \frac{\partial}{\partial x} \theta_{4}\left(\frac{x}{2 l} \mid \frac{i \pi t}{l^{2}}\right)$

$L^{-1}[\operatorname{csch}(l \sqrt{p})]=-\frac{1}{l} \frac{\partial}{\partial x} \theta_{4}\left(\frac{x}{2 l} \mid \frac{i \pi t}{l^{2}}\right) ; x=0$

As Equações 36 e 37 envolvem a função de Jacobi Theta do tipo 4 , representada por $\theta_{4}$. A definição dessa função é dada pela Equação 38.

$\theta_{4}(v \mid \tau)=(-i \tau)^{\frac{-1}{2}}\left[\sum_{n=0}^{\infty} e^{\frac{-i \pi\left(v+\frac{1}{2}+n\right)^{2}}{\tau}}-\sum_{n=-1}^{-\infty} e^{\frac{-i \pi\left(v+\frac{1}{2}+n\right)^{2}}{\tau}}\right]$

A aplicação da definição de Jacobi Theta nas Equações 36 e 37, levas as seguintes expressões.

$L^{-1}\left[\frac{\cosh (x \sqrt{s})}{\operatorname{senh}(l \sqrt{s})}\right]=\frac{l}{\sqrt{\pi} t^{\frac{3}{2}}} \sum_{n=0}^{\infty}\left(\frac{x}{2 l}+\frac{1}{2}+n\right) e^{\frac{-l^{2}}{t}\left(\frac{x}{2 l}+\frac{1}{2}+n\right)^{2}}$

$\frac{-l}{\sqrt{\pi} t^{\frac{3}{2}}} \sum_{n=-1}^{-\infty}\left(\frac{x}{2 l}+\frac{1}{2}+n\right) e^{\frac{-l^{2}}{t}\left(\frac{x}{2 l}+\frac{1}{2}+n\right)^{2}}$

$L^{-1}[\operatorname{csch}(l \sqrt{p})]=\frac{2 l}{\sqrt{\pi} t^{\frac{3}{2}}} \sum_{n=0}^{\infty}\left(n+\frac{1}{2}\right) e^{\frac{-l^{2}}{t}\left(n+\frac{1}{2}\right)^{2}}$
Pela substituição dos parâmetros da Equação 33 nas Equações 39 e 40, encontra-se que:

$L^{-1}\left[\frac{\cosh \left(L \sqrt{\frac{S}{D}}\right)}{\operatorname{senh}\left(\frac{3 L}{2} \sqrt{\frac{S}{D}}\right)}\right]=\frac{3 L}{2 \sqrt{\pi D} t^{\frac{3}{2}}} \sum_{n=0}^{\infty}\left(n+\frac{5}{6}\right) e^{\frac{-9 L^{2}}{4 t \sqrt{D}}\left(n+\frac{5}{6}\right)^{2}}$
$+\frac{3 L}{2 \sqrt{\pi D} t^{\frac{3}{2}}} \sum_{n=1}^{\infty}\left(n-\frac{5}{6}\right) e^{\frac{-9 L^{2}}{4 t \sqrt{D}}\left(n-\frac{5}{6}\right)^{2}}$

$L^{-1}\left[\operatorname{csch}\left(\frac{L}{2} \sqrt{\frac{S}{D}}\right)\right]=\frac{L}{\sqrt{\pi D} t^{\frac{3}{2}}} \sum_{n=0}^{\infty}\left(n+\frac{1}{2}\right) e^{\frac{-L^{2}}{4 D t}\left(n+\frac{1}{2}\right)^{2}}$

Através da aplicação dos resultados das Equações 41 e 42 no teorema da convolução, Equação 35. Encontra-se que a função $h(t)$ é representada pela expressão seguinte.

$$
\begin{aligned}
& h(t)=\frac{3 L^{2}}{2 \pi D} \int_{0}^{t} \frac{1}{\tau^{\frac{3}{2}}(t-\tau)^{\frac{3}{2}}} \sum_{n=0}^{\infty}\left(n+\frac{1}{2}\right) e^{\frac{-L^{2}}{4 D(t-\tau)}\left(n+\frac{1}{2}\right)^{2}} \\
& \left(\sum_{n=0}^{\infty}\left(n+\frac{5}{6}\right) e^{\frac{-9 L^{2}}{4 \tau \sqrt{D}}\left(n+\frac{5}{6}\right)^{2}}+\sum_{n=1}^{\infty}\left(n-\frac{5}{6}\right) e^{\frac{-9 L^{2}}{4 \tau \sqrt{D}}\left(n-\frac{5}{6}\right)^{2}}\right) d \tau
\end{aligned}
$$

$\mathrm{Na}$ busca por um estado estacionário do sistema, deseja-se definir o momento em que o valor da função $h(t)$ será fixo com o passar do tempo. Nesse caso, o fluxo na superfície absorvente será constante e igual ao deslocado para a posição $\frac{L}{2}$. Devido a esse fato, adota-se que a partir de um determinado tempo $\varepsilon$ esse estado estacionário será atingido. Nesse instante de tempo, a função $h(t)$ pode deixar o integrando da Equação 26, como apresenta a Equação seguinte.

$C_{1}(x, t)=C(x, t)+h(\epsilon) \int_{\epsilon}^{t} C\left(x, t-t^{\prime}\right) d t^{\prime}, t>\epsilon$

Quanto menor o tamanho do anel e maior for a difusividade dos animais, mais rápido será o tempo de estabilização. Dessa forma, considera-se o caso em que este tempo seja muito pequeno, de modo que $\varepsilon \rightarrow 0$. Consequentemente, a Equação 44 passa a ser escrita com o uso de limites.

$$
C_{1}(x, t)=C(x, t)+\left(\lim _{\epsilon \rightarrow 0} h(\epsilon)\right)\left(\lim _{\epsilon \rightarrow 0} \int_{\epsilon}^{t} C\left(x, t-t^{\prime}\right) d t^{\prime}\right)
$$


A partir da utilização da Equação 17, encontra-se o valor da integração realizada no lado esquerdo da Equação 45. Para este cálculo foi realizada uma adaptação para que o reinício ocorresse para a metade da distância máxima possível entre os caminhantes. Diferentemente da situação em que ela foi obtida, na qual o reinício ocorria para a distância máxima permitida. O resultado da integração é dado pela Equação 46

$\int_{0}^{\infty} C\left(x, t-t^{\prime}\right) d t^{\prime}$

$=\sum_{n=1}^{\infty} \frac{4 L}{(2 n-1)^{2} \pi^{2} D} \operatorname{sen}\left(\frac{(2 n-1) \pi}{4}\right) \operatorname{sen}\left(\frac{(2 n-1) \pi x}{L}\right)$

A definição do valor estacionário para a função $h(t)$ é realizada pela expansão da função $h(s)$ em séries de potências. E logo em seguida utiliza-se o teorema do valor final (Boyce e Diprima, 2010).

$h(s)=\frac{2 D}{3 L^{2} s}+\frac{1}{18}-\frac{37 L^{2} s}{1080 D}+\frac{83 L^{4} s^{2}}{9072 D^{2}}-\cdots$

$\lim _{t \rightarrow \infty} h(t)=\lim _{s \rightarrow 0} s \cdot h(s)=\frac{2 D}{3 L^{2}}$

Este resultado foi obtido para um sistema de tamanho 2L, como enunciado antes de aplicar o método de imagem. Ao realizar a modificação para um sistema de tamanho $\frac{L}{2}$, caso solucionado pelo método de separação de variáveis, tem-se que:

$\lim _{t \rightarrow \infty} h(t)=\frac{32 D}{3 L^{2}}$

Com posse das Equações 46 e 49, encontra-se a expressão para $C_{l}(x)$, que equivale a $C_{l}(x, t)$ no estado estacionário.

$$
\begin{aligned}
& C_{1}(x)=\lim _{t \rightarrow \infty} h(t) \int_{0}^{\infty} C(x, u) d u \\
& =\sum_{n=1}^{\infty} \frac{132}{3 L(2 n-1)^{2} \pi^{2}} \operatorname{sen}\left(\frac{(2 n-1) \pi}{4}\right) \operatorname{sen}\left(\frac{(2 n-1) \pi x}{L}\right)
\end{aligned}
$$

Através da comparação entre as Equações 45 e 50, verifica-se a ausência da contribuição do termo $C(x, t)$ no membro central da Equação 50. Esse fato se deve a tendência de não existirem trajetórias que não sofreram reinício quando o tempo tende ao infinito. Matematicamente, este fato pode ser representa pela seguinte expressão. $\lim _{t \rightarrow \infty} C(x, t)=0$

Com posse da Equação 50, nota-se que o estado estacionário independe da constante de difusão, como era de se esperar. Dessa maneira, essa variável influencia apenas em quão rápido o sistema chega a esse estado de equilíbrio. A representação do estado estacionário é apresentada graficamente pela figura 4 .

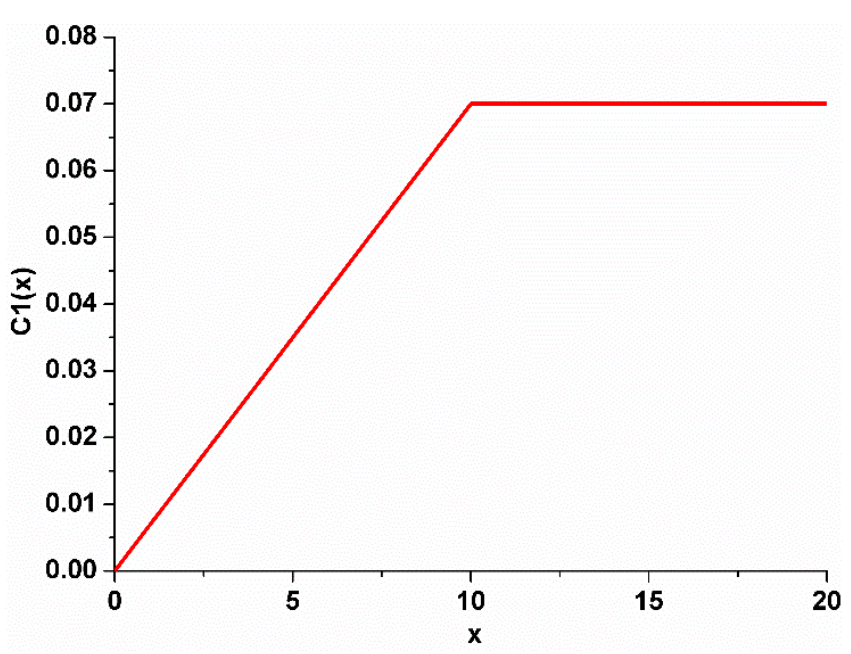

Figura 4 -Função densidade de probabilidade representativa do estado estacionário do sistema.

Com esta informação finaliza-se o estudo do caminhante imortal, restando uma análise numérica para o caso mortal. Optou-se pela análise numérica devido ao fato da dificuldade de trabalhar com esse novo parâmetro de forma analítica.

\subsection{Análise do sistema para o caminhante mortal}

Esta subseção realiza um estudo numérico sobre um caminhante mortal a procura de alimento com a mesmas características da subseção anterior. De modo que a caminhada se inicia na metade da distância máxima permitida e há o processo de reinício para essa posição após o encontro da presa e do predador. Dessa forma, desenvolvese um gráfico que apresenta o número médio de passos executados por um caminhante antes de morrer. Esse número médio foi obtido através da quantificação de 100 caminhadas sob as mesmas condições. Este número médio de passes trata-se de uma função da taxa metabólica $S$ preestabelecida. De forma que o predador não pode realizar mais do que $S$ passos sem se alimentar.

A Figura 5 é uma representação para um anel com um total de 40 sítios e com os caminhantes a uma distância inicial de 10 sítios. 


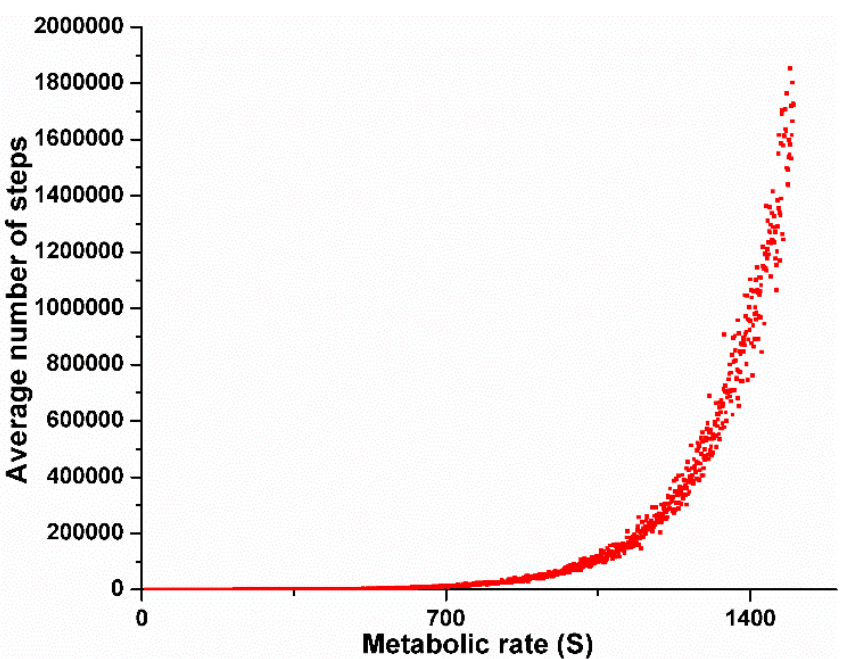

Figura 5 -Número de passos de um caminhante em função da sua taxa metabólica.

Com base na Figura 5, pode-se notar um crescimento abrupto do número de passos do caminhante com o aumento da sua taxa metabólica. Dessa forma, podese concluir que um pequeno aumento na capacidade metabólica leva a um grande aumento na capacidade de sobreviver. Após a realização de ajustes para identificar a melhor equação que representa essa curva, obteve-se o melhor resultado com a função exponencial. Esta relação apresentou um coeficiente de correlação $\left(R^{2}\right)$ igual a 0.975 , os parâmetros ótimos que levam a este ajuste estão representados na Equação 52. Nesta equação, a variável $y$ representa o número médio de passos do caminhante até sua morte e $S$ trata de sua capacidade metabólica.

$y=180.8492 \cdot e^{0.0061 \cdot S}$

\section{CONCLUSÕES}

Este artigo apresentou resultados analíticos e numéricos até então inéditos para a área de processos estocásticos. Dessa maneira, contribuiu para que futuros pesquisadores de sistemas similares tenham conhecimento de como abordar esses problemas. Este trabalho encontrou algumas características do sistema estudado, entre elas temse a indiferença do estado de equilíbrio quanto a velocidade dos caminhantes. Esta característica pode ser associada diretamente com o equilíbrio de reações químicas, de forma que o estado de equilíbrio é influenciado apenas pelas condições termodinâmicas e não pela cinética da reação. Este estudo não finalizou todas abordagens possíveis desses sistemas, sendo ainda possível o desenvolvimento de estudos para abordar configurações com diversos caminhantes ou fontes de alimento. Dessa forma, este artigo trata-se de uma porta de entrada para novos avanços na área.

\section{AGRADECIMENTOS}

Os autores agradecem o apoio por parte da Universidade Federal de São João Del-rei (UFSJ) e da
Universidade Federal de Viçosa (UFV). O presente trabalho foi realizado com o apoio da Coordenação de Aperfeiçoamento de Pessoal de Nível Superior - Brasil (CAPES) - Código de Financiamento 001 e da Fundação de Amparo à Pesquisa do Estado de Minas Gerais (FAPEMIG).

\section{R E F E R E N C I A S}

BELAN S. Restart could optimize the probability of success in a Bernoulli trial. Physical Review Letters, v. 120, 080601, feb. 2018.

BOYCE, W.; DIPRIMA, R. Elementary Differential Equations and Boundary Value Problems, $9^{\text {th }}$ ed., New York: LTC, 2010.

BROWN, R. A brief account of microscopical observations made in the months of June, July and August 1827, on the particles contained in the pollen of plants; and on the general existence of active molecules in organic and inorganic bodies. Philosophical Magazine, v.4, n. 21, p. 161-173, sep. 1828.

CHUPEAU, M.; BÉNICHOU, O.; REDNER, S. Universality classes of foraging with resource renewal. Physics Review E, v.93, n. 6, mar. 2016.

EINSTEIN, A. On the motion of small particles suspended in liquids at rest required by the molecular-kinetic theory on heat. Annalen der Physik, v.17, p. 549-560, may 1905.

ERDÉLYI, A. Tables of integral transforms, v.1. $1^{\text {st }}$ ed., New York: McGraw-Hill Book Company, 1954.

EVANS, M.; MAJUMDAR, S. Diffusion with Optimal Resetting. Journal of Physics A: Mathematical and Theoretical, v. 44, n.43, oct. 2011.

FALCÃO, R.; EVANS, M. Interacting Brownian Motion with Resetting. Journal of Statistical Mechanics: Theory and Experiment, v. 2017, feb. 2017.

FISHER, M. Walks, Walls, Wetting and Melting. Journal of Statistical Physics, v. 34, n. 5, oct. 1984.

GABEL, A.; MAJUMDAR, S.; PANDURANGA N.; REDNER S. Can a Lamb Reach a Haven Before Being Eaten by Diffusing Lions? Journal of Statistical Mechanics: Theory and Experiment, v. 2012, may 2012.

GOUY, L. Note sur le mouvement brownien. J. Phys. Theor. Appl., v.7, n. 1, p. 561-564, 1888.

HSU, H. Probability, random variables and stochastic processes. $3^{\text {th }}$ ed., New York: McGraw-Hill, 2014.

LUTZER, C. De-mystifying the Dirac $\delta$-function. Primus: Problems, Resources, and Issues in Mathematics Undergraduate Studies, v. 17, n.4, p. 373-377, 2007.

MARQUES, F.; CHAUÍ-BERLINK J. Modelos de estratégia de busca: será o passeio de Lévy a solução? Revista da Biologia, v. 12, n. 1, jul. 2014.

PTASZYNSKI, K. First-passage times in renewal and nonrenewal systems. Phys. Rev. E, v. 97, 012127, jan. 2018.

REDNER, S. A guide to first-passage processes. $1^{\text {st }}$ ed., Cambridge: Cambridge University Press, 2001.

REDNER, S; BÉNICHOU, O. Gradual diffusive capture: slow death by many mosquito bites. Journal of Statistical Mechanics: Theory and Experiment, v.2014, P. 11019, nov. 2014. 
SOKOLOV, I.; KLAFTER, J. From diffusion to anomalous diffusion: A century after Einstein's Brownian motion. Chaos: An Interdisciplinary Journal of Nonlinear Science, v. 15, n. 2, 026103, jun. 2005.

SMITH, S.; GRIMA, R. An exact solution to Brownian dynamics of a reversible bimolecular reaction in one dimension. Preprinter arXiv:1605.05557v1, May 2016. Available at: https://arxiv.org/abs/1605.05557>. Accessed on
November $14^{\text {th }}, 2017$.

STEWART, J. Cálculo, v. 2. $8^{\mathrm{a}}$ ed., São Paulo: CENGAGE Learning, 2017.

WIDDER, D. The Heat Equation. $1^{\text {st }}$ ed., New York: Academic Press, 1975.

WILF, H. Generatingfunctionology. $2^{\text {nd }}$ ed., Philadelphia: Academic Press, 1992. 